\title{
Mit reguliertem Wettbewerb zu mehr Qualität und Kosteneffizienz im Gesundheitswesen
}

\author{
Die Arbeitsgruppe Forum Santé-Gesundheit macht sich seit 10 Jahren für \\ ein nachhaltiges Schweizer Gesundheitswesen stark. An ihrer 10. Tagung am \\ 17. Januar 2008 in Bern hat das multidisziplinäre Gremium seine Vorschläge \\ für die Zukunft der Gesundheitspolitik der Öffentlichkeit vorgestellt.
}

Winfried Suske

\footnotetext{
* Herkunft der Tagungsteilnehmer nach Branchen: 19,8\% Behörden, $11,3 \%$ Versicherungen, $14,1 \%$ Industrie, 24,9\% Medizin/Pflege, 13,0\% Apotheken, 16,9\% andere.
}

Korrespondenz:

Dr. Winfried Suske

Dr. Schlegel Healthworld AG

CH-6312 Steinhausen

Tel. 0417487629

Fax 0417487611

w.suske@schlegelhealth.ch

www.forumgesundheit.ch
Die neuesten Empfehlungen der Arbeitsgruppe drehen sich um eine gerechte Verteilung der Ressourcen durch die öffentliche Hand, um Transparenz und um die Finanzierung des Gesundheitswesens. Weitere Schwerpunkte bilden die Information und Aufklärung der Patienten oder die Eigenverantwortung der Versicherten, was zum Beispiel ihren Lebensstil und krankheitsvorbeugende Massnahmen betrifft.

Alle Vorschläge des Forums Santé-Gesundheit zielen darauf $a b$, eine qualitativ hochwertige medizinische und pflegerische Versorgung der Bevölkerung zu einem möglichst günstigen Preis zu garantieren - mit Chancengleichheit und Wahlfreiheit als wichtigen Grundpfeilern.

Rund 90\% der über 250 Tagungsteilnehmer* erklärten sich bei der Abstimmung zum Thema Ressourcensteuerung damit einverstanden, dass der Bund im Rahmen des Krankenversicherungsgesetzes (KVG) ein Mandat zur Lenkung der finanziellen Mittel und der Versorgungskapazitäten erhalten solle. Und zwar nicht nur bei den Massnahmen zur Prävention und zur Gesundheitsförderung, sondern auch beim kurativen und medizinischen Angebot. Dies würde eine Abkehr vom bisherigen Prinzip bedeuten, bei dem die Kantone den Leistungsauftrag für die Gesundheitsversorgung der Bürger besitzen und sich mit dem Bund abstimmen.

Die vom Forum Santé-Gesundheit postulierte Regulatorrolle des Bundes erhielt an der Tagung auch am traditionellen runden Tisch eine breite Unterstützung. Über die Vorschläge diskutierten der Ständerat und Präventivmediziner Prof. Felix Gutzwiller, der Direktor des Dachverbands der schweizerischen Krankenversicherer (santésuisse) Fritz Britt, der Nationalrat und Vizepräsident des Dachverbandes Schweizerischer Patientenstellen Jean-François Steiert und der Genfer Regierungsrat und Gesundheitsdirektor Pierre-François Unger.
Nach dem Modell des regulierten Wettbewerbs solle der Bund den ordnungspolitischen Rahmen bzw. die Spielregeln für den Wettbewerb zwischen den Versicherern und den Leistungserbringern vorgeben, ohne mehr Macht an die Krankenversicherungen $\mathrm{zu}$ delegieren, lautete die einhellige Meinung. Dabei müssen die Qualität und die Breite der medizinischen Versorgung, die chancengerechte Zugänglichkeit und die Transparenz bezüglich der erbrachten Leistungen im Vordergrund stehen.

Unangetastet bleiben sollen im Falle einer Umsetzung die Vielfalt der Versicherungsmodelle - diese werden künftig durch interdisziplinäre Versorgungsnetzwerke und ManagedCare-Modelle ergänzt - und die Wahlfreiheit der Patienten.

Ungeteilte Zustimmung fanden auch die Empfehlungen des Forums Santé-Gesundheit, die Wirtschaftlichkeit des Gesundheitssystems mit Hilfe regionaler und nationaler Indikatoren $\mathrm{zu}$ messen und, als überfällige Massnahme, Daten über die Kosten und die Wirksamkeit der erbrachten Leistungen verfügbar zu machen. Fritz Britt bezeichnete es in diesem Zusammenhang als unhaltbar, dass keine offizielle Stelle in der Schweiz über vergleichbare Kenndaten zu den Gesundheitsleistungen verfüge. Auf den Missstand einer fehlenden Gesamtdatentransparenz weist auch der 2006 veröffentlichte Bericht von OECD und WHO zum Schweizer Gesundheitssystem hin. Dessen Hauptautorin, Francesca Colombo, trat als Gastrednerin an der Tagung in Bern auf.

Die Herausgabe vergleichbarer Daten durch die ambulanten und stationären Leistungsträger müsse der Bund, wenn nötig, erzwingen, forderte Fritz Britt. In Verbindung mit der gebotenen Transparenz sind die beschlossenen Fallpauschalen bei der Spitalfinanzierung ein Schritt in Richtung einer verbesserten Effizienz des Gesundheitssystems. 
Die zentrale Bedeutung einer globalen Sicht auf die Gesundheit - diese wird nicht zuletzt von OECD und WHO gefordert - brachte der Präventivmediziner Felix Gutzwiller gezielt auf den Punkt. «Leider haben wir in der Schweiz keine explizite Gesundheitspolitik, sondern betreiben im Prinzip eine Krankenversicherungspolitik. Wir brauchen statt dessen endlich eine öffentliche Debatte darüber, welche Leistungen die Gesundheit der Bevölkerung benötigt und welche Faktoren ihre Gesundheit beeinflussen.»

Als Basis für eine solche Debatte, wie sie das Forum Santé-Gesundheit seit Jahren anregt, wäre laut Gutzwiller eine formulierte Gesundheitspolitik hilfreich, die auf die Prävention und damit gleichzeitig auf mehr Eigenverantwortung der Versicherten setzt. Während hierzulande Milliarden in die Behandlung von Wohlstandskrankheiten wie Diabetes und Fettleibigkeit gepumpt würden, gebe die Schweiz für Präventionsmassnahmen nur 2,2\% der gesamten Gesundheitskosten von geschätzten 52 Mrd. Franken aus. Abhilfe schaffen könnte das geplante Präventionsgesetz, in dem die nationalen Präventions- und Gesundheitsförderungsziele verankert werden sollen. Gemäss Gutzwiller liegen die Eckdaten für das neue eidgenössische Gesetz, für das das Departement Couchepin bis Herbst 2008 einen Vorentwurf erarbeitet, bereits vor.

Die 11. Tagung des Forum Santé-Gesundheit soll am 22. Januar 2009 in Montreux stattfinden. Bis dahin soll es gelingen, auch Vertreter der Politik in die ehrenamtlich tätige Arbeitsgruppe einzubinden, um die Diskussion über Gesundheitsziele auf noch breiterer Basis in Gang zu bringen.
Es folgen die Abstimmungsergebnisse im einzelnen:

\section{Ressourcensteuerung}

These 1.1: Erteilen eines umfassenden Mandats an den Bund zur Steuerung der Massnahmen für Prävention, für Gesundheitsförderung, für kurative und medizinische Angebote.

- sehr einverstanden: 50,5\%;

- einverstanden: 37,9\%;

- nicht einverstanden: 7,1\%;

- gar nicht einverstanden: 3,8\%;

- weiss nicht: $0,5 \%$.

These 1.2: Die Steuerung der Massnahmen für Prävention, für Gesundheitsförderung, für kurative und medizinische Angebote muss zwischen Bund und Kantonen koordiniert werden. Die Kantone sind zuständig für die regionale Versorgung und die Nahversorgung, der Bund für die Spitzenmedizin und die universitäre Medizin.

- sehr einverstanden: 44,6\%;

- einverstanden: 32,5\%;

- nicht einverstanden: 13,9\%;

- gar nicht einverstanden: 8,4\%;

- weiss nicht: $0,6 \%$.

\section{Effizienzsteigerung (eine Wahl)}

- Einführung von Ärztenetzwerken und Netzwerken anderer Leistungserbringer als eigentliche zentrale Referenzmodelle: 43,2\%;

- Beibehaltung der klassischen Form der obligatorischen Krankenversicherung mit Netzwerken als speziellen Versicherungsformen (Status quo): 11,2\%;

- Beibehaltung des Status quo, aber neue Ausrichtung der Ausbildung des medizinischen

langfristigen, für alle akzeptablen Lösungen sowie 4. Entwicklung eines Gesundheitskonzepts, das den Patienten in den Mittelpunkt stellt. An den jährlich stattfindenden Tagungen stellt Forum Santé-Gesundheit seinen Bericht und seine Empfehlungen einem breiten Publikum vor. Es verschafft sich ausserdem bei den Entscheidungsträgern und den Playern des Gesundheitswesens Gehör mit seinen Newsletters und Publikationen «Für eine qualitativ hochwertige Gesundheitsversorgung in der Schweiz». Weitere Informationen sind der Website www.forumgesund heit.ch zu entnehmen. Die Arbeitsgruppe wird von Sanofi-Aventis (Schweiz) AG und der Universität Genf logistisch unterstützt. 
Personals auf interdisziplinäre Zusammenarbeit und Aufwertung des Pflegeberufes: $45,6 \%$.

\section{Transparenz (eine Wahl)}

- Entwicklung nationaler und regionaler Indikatoren zur Messung der Wirksamkeit und Wirtschaftlichkeit des Gesundheitssystems: $68,3 \%$;

- Zugänglichmachen von vergleichbaren Daten über die Sicherheit, Wirksamkeit und Kosten der Behandlungen in Spitälern: $17,6 \%$;

- Zugänglichmachen von vergleichbaren Daten über die Sicherheit, Wirksamkeit und Kosten ambulanter Leistungen: 14,1\%.

\section{Patienteninformation (eine Wahl)}

- Schaffung eines umfassenden nationalen Gesundheitsführers: 26,6\%;

- Evaluation und Zertifizierung von Internetseiten zu medizinischen Fragen: 10,1\%;

- strenge Trennung von Werbung und Information bei medizinischen und pharmazeutischen Angeboten: 25,4\%;

- Förderung von Gesundheitsnetzwerken und systematische Einführung von Gesundheitsberatern: $37,9 \%$.

\section{Patientenverantwortung (eine Wahl)}

- Erlass eines eidgenössischen Präventionsgesetzes: $29,1 \%$;

- Einführung der Versichertenkarte und elektronischer Patientendossiers: 43,6\%;

- Einführung einer eigentlichen Präventionssteuer auf belastenden Produkten (Alkohol, Tabak, Produkte mit hohen Fett- oder Zuckeranteilen usw.): 27,3\%.

\section{Finanzierung (zwei Wahlmöglichkeiten)}

- Reduktion des Leistungskatalogs in der Grundversicherung: 7,8\%;

- Spitalfinanzierung mittels Fallpauschale: $19,4 \%$;

- Erhöhung der Bundesbeiträge auf das Niveau der 80er Jahre: 7,5\%;

- zusätzliche Belastung der Personen ab 65 mit hohem Vermögen bzw. Einkommen: 4,4\%;

- Wettbewerb unter den Krankenversicherungen ohne Risikoselektion: 11,9\%;

- Rationierung teurer Angebote: 3,7\%;

- Verbesserung der Kohärenz medizinischer Leistungen: 27,2\%;

- ständige Kontrolle von Angebot und Nachfrage und öffentliche Diskussion der Ergebnisse: $18,0 \%$. 